\title{
The MAP Kinase Pmk1 and Protein Kinase A Are Required for Rotenone Resistance in the Fission Yeast, Schizosaccharomyces pombe
}

\author{
Yiwei Wang ${ }^{1}$, Galina Gulis ${ }^{1}$, Scott Buckner ${ }^{1}$, P. Connor Johnson ${ }^{1}$, Daniel Sullivan ${ }^{1}$, Laura \\ Busenlehner $^{2}$, and Stevan Marcus s $^{1,3}$ \\ ${ }^{1}$ Department of Biological Sciences, The University of Alabama, Tuscaloosa, AL 35487 \\ ${ }^{2}$ Department of Chemistry, The University of Alabama, Tuscaloosa, AL 35487
}

\begin{abstract}
Rotenone is a widely used pesticide that induces Parkinson's disease-like symptoms in rats and death of dopaminergic neurons in culture. Although rotenone is a potent inhibitor of complex I of the mitochondrial electron transport chain, it can induce death of dopaminergic neurons independently of complex I inhibition. Here we describe effects of rotenone in the fission yeast, Schizosaccharomyces pombe, which lacks complex I and carries out rotenone-insensitive cellular respiration. We show that rotenone induces generation of reactive oxygen species (ROS) as well as fragmentation of mitochondrial networks in treated S. pombe cells. While rotenone is only modestly inhibitory to growth of wild type $S$. pombe cells, it is strongly inhibitory to growth of mutants lacking the ERK-type MAP kinase, Pmk1, or protein kinase A (PKA). In contrast, cells lacking the p38 MAP kinase, Spc1, exhibit modest resistance to rotenone. Consistent with these findings, we provide evidence that Pmk1 and PKA, but not Spc1, are required for clearance of ROS in rotenone treated $S$. pombe cells. Our results demonstrate the usefulness of $S$. pombe for elucidating complex I-independent molecular targets of rotenone as well as mechanisms conferring resistance to the toxin.
\end{abstract}

\section{Introduction}

Rotenone is a five-ring isoflavonoid produced naturally by a number of different plant species, in particular of the genera Lonchocarpus and Derris [1]. Rotenone exhibits strong pesticidal and piscicidal activities, which have been attributed to its potency as an inhibitor of complex I of the mitochondrial electron transport chain [2;3], a large membrane-spanning NADH-ubiquinone oxidoreductase enzyme complex consisting of more than forty distinct polypeptide subunits [4]. In addition to its inhibitory effects on complex I, rotenone has been shown to induce depolymerization of microtubules in some types of cultured mammalian cells and to inhibit assembly of microtubules in vitro, possibly through direct interaction with tubulin [5;6].

\footnotetext{
(C) 2010 Elsevier Inc. All rights reserved.

${ }^{3}$ Address correspondence to: Stevan Marcus, Dept. of Biological Sciences, The University of Alabama, Department of Biological Sciences, Box 870344, Tuscaloosa, AL 35487, smarcus@bama.ua.edu.

Publisher's Disclaimer: This is a PDF file of an unedited manuscript that has been accepted for publication. As a service to our customers we are providing this early version of the manuscript. The manuscript will undergo copyediting, typesetting, and review of the resulting proof before it is published in its final citable form. Please note that during the production process errors may be discovered which could affect the content, and all legal disclaimers that apply to the journal pertain.
} 
Earlier this decade, it was reported that rats chronically treated with rotenone develop behavioral symptoms and pathophysiologies similar to those characteristic of Parkinson's disease (PD), including motor defects and dopaminergic neuron degeneration, respectively [7]. These findings fueled speculation that pesticides and other environmental toxins might contribute to the development of PD [8]. They also provided support for the notion that complex I inhibition might be contributory to the pathogenesis of PD, a speculation raised several years earlier as a result of postmortem analyses of PD patients, which revealed evidence of complex I deficiency and oxidative stress damage in the substantia nigra region of the brain $[9 ; 10 ; 11]$, and from the discovery that drug abusers inadvertently exposed to the complex I inhibitor, 1-methyl-4-phenylpyridinium $\left(\mathrm{MPP}^{+}\right)$, developed clinical PD [12].

Despite substantial correlative data, a direct causal link between complex I inhibition and PD has yet to be established. Indeed, Choi et al. [13] recently showed that dopaminergic neurons from $\mathrm{Ndufs} 4^{--}$mutant mouse embryos lacking detectable complex I activity are actually substantially more sensitive to rotenone-induced cell death than neurons from wild type mouse embryos. In addition, these investigators reported that mutant adult mice in which complex I was selectively inactivated in cells expressing tyrosine hydroxylase (the rate-limiting enzyme in dopamine synthesis) did not develop behavioral or biochemical defects associated with PD. These findings demonstrate that rotenone-induced dopaminergic cell death is not fully accounted for by complex I inhibition and that complex I inhibition alone, at least in mice, is insufficient to induce PD.

In light of the discovery that rotenone is highly toxic to dopaminergic neurons lacking complex I activity, it is worthwhile to reassess whether new insights regarding molecular targets of and physiological responses to the toxin might be gained from investigations using model organisms that lack complex I of the mitochondrial electron transport chain. In this regard, the evolutionarily distant yeasts Schizosaccharomyces pombe (fission yeast) and Saccharomyces cerevisiae (budding yeast) have each lost the genes encoding complex I subunits during the course of evolution and have been shown to carry out rotenone insensitive cellular respiration $[14 ; 15 ; 16]$. In the present study, we show that while rotenone has no discernable effect on cellular respiration in S. pombe, it nevertheless induces generation of reactive oxygen species (ROS) and disruption of tubular mitochondrial networks in treated $S$. pombe cells. In addition, we show that while rotenone is only modestly inhibitory to growth of wild type $S$. pombe cells, it is profoundly inhibitory to mutants lacking the ERK-type mitogen-activated kinase (MAPK) homolog Pmk1 (a.k.a. Spm1)[17;18] or protein kinase A (PKA). Lastly, we provide evidence that Pmk1 is required for normal clearance of ROS in rotenone-treated $S$. pombe cells and that PKA plays a role in ROS clearance even under normal growth conditions.

\section{Materials and methods}

\section{Yeast strains, media, and genetic methods}

S. pombe strains used in this study were SP870 ( $h^{90}$ ade6-210 leul-32 ura4-D18), SPSPC1U90 ( $h^{90}$ spc14::ura4 ade6-210 leu1-32 ura4-D18), SPM1L ( $h^{90}$ spm14::LEU2 leu1-32 ura4-D18), and CHP453 ( ${ }^{-}$pkal4 ura4 leul-32 his7-366 ura4-D18). S. pombe cultures were grown in YES medium (0.5\% yeast extract; $3 \%$ dextrose; and adenine, histidine, leucine, lysine and uracil, each at $250 \mathrm{mg} / \mathrm{L}$ )[19]. Agar media contained 2\% Bacto agar (Difco). Rotenone stock solutions (10 mM in DMSO) were prepared just prior to carrying out each experiment. Rotenone containing growth media were prepared by mixing rotenone stock solution with YES medium as required for the desired rotenone concentration (up to $6.4 \mu \mathrm{g} / \mathrm{ml}$ ). For agar media, rotenone stock solution was added after autoclaving and cooling of the media to approximately $45^{\circ} \mathrm{C}$. For all experiments, DMSO was added to control media at concentrations equivalent to that in media supplemented with rotenone. 


\section{MitoTracker Green FM and dihydroethidium staining of S. pombe cells}

Mitochondrial localization was detected by staining S. pombe cells with MitoTracker Green FM (MTGreen) (Invitrogen). Briefly, S. pombe cells were cultured overnight in YES liquid medium at $30^{\circ} \mathrm{C}$ to mid-log phase. Rotenone stock solution (see above) was added to portions of each culture to a final concentration of $6.4 \mu \mathrm{g} / \mathrm{ml}$. Equivalent volumes of DMSO were added to control cultures. Staining was carried out by adding $10 \mu \mathrm{l}$ of MTGreen stock solution ( $10 \mu \mathrm{M}$ in DMSO) to $1 \mathrm{ml}$ of cell culture and incubating at $30^{\circ} \mathrm{C}$ with shaking for $20 \mathrm{~min}$. Stained cells were washed once with YES and resuspended in approximately $10 \mu \mathrm{l}$ of YES prior to preparation of samples for microscopy. Dihydroethidium (DHE) staining was carried out as described [20]. Stained cells were visualized by epifluorescence microscopy using a Nikon 90i epifluorescence microscope system equipped with a CoolSNAP HQ2 monochrome CCD camera (Photometrics).

\section{Respiration assays}

Whole-cell respiration rates were measured using a Strathkelvin Model 782 dissolved oxygen measuring system equipped with a Clark-type microcathode oxygen electrode (Strathkelvin Instruments Limited, North Lanarkshire, Scotland). S. pombe cells were cultured in YES medium at $23^{\circ} \mathrm{C}$ to a density of $2-3 \times 10^{6}$ cells $/ \mathrm{ml}$. Eight $\mu 1$ of DMSO or DMSO containing $10 \mathrm{mM}$ rotenone was added to $5 \mathrm{ml}$ portions of cell culture, which were incubated for $3 \mathrm{hr}$ at $23^{\circ} \mathrm{C}$. One $\mathrm{ml}$ of each sample was used for measurement of oxygen consumption and the remainder of the culture was used for spectrophotometric determination of cell density (OD $600 \mathrm{~nm}$ ). Duplicate cultures were used for each experiment.

\section{Results}

\section{Effects of rotenone on S. pombe cell growth and respiration}

We initiated this investigation by carrying out experiments to confirm findings of a previous study showing that rotenone has negligible effects on $S$. pombe cell growth and respiration [16]. To do this, we prepared YES agar medium containing concentrations of rotenone ranging from 0.2 to $6.4 \mu \mathrm{g} / \mathrm{ml}$ (Materials and Methods). While this range of concentration is higher than that required for inhibition of respiration by rotenone in cultured mammalian cells [21], we sought to take into account the possibility that cellular penetrance of rotenone might be limited by the $S$. pombe cell wall as well as the possibility that the toxin might be more rapidly metabolized in $S$. pombe cells than in animal cells. Mid-log phase cultures of wild type $S$. pombe cells were serially diluted and tested for growth by spot assay on medium containing rotenone and on control medium lacking the toxin. Marked inhibition of S. pombe growth was detected on medium containing $6.4 \mu \mathrm{g} / \mathrm{ml}$ rotenone but not on media containing lower concentrations of the toxin (Fig. 1A). To confirm that rotenone has no effect on cellular respiration in $S$. pombe, wild type $S$. pombe cells were cultured to mid-log phase, then treated, or not, for $3 \mathrm{hr}$ with rotenone $(6.4 \mu \mathrm{g} / \mathrm{ml})$ or the mitochondrial complex III inhibitor antimycin A $(1 \mu \mathrm{g} / \mathrm{ml})$. Rates of oxygen consumption were then measured in toxin-treated and control cultures. As shown in Fig. 1B, rotenone did not inhibit S. pombe respiration, whereas treatment with antimycin A resulted in a greater than 10-fold reduction in the rate of respiration. These results indicate that at the maximum concentration tested $(6.4 \mu \mathrm{g} / \mathrm{ml})$, rotenone partially inhibits $S$. pombe cell growth without measurably affecting cellular respiration. 
Rotenone induces generation of ROS and fragmentation of mitochondrial networks in S. pombe cells

Since rotenone induces generation of ROS in cultured mammalian cells and oxidative stress damage in the brains of rats chronically treated with the toxin [22;23], we sought to determine whether it also induces generation of ROS in S. pombe cells. To do this, we treated wild type $S$. pombe cells with rotenone $(6.4 \mu \mathrm{g} / \mathrm{ml})$ and periodically examined the treated cells and cells from control cultures by staining with dihydroethidium (DHE), which is oxidized to the fluorescent compound ethidium in the presence of ROS [24]. S. pombe cells treated with rotenone for $6 \mathrm{hrs}$ (Fig. 2A, panel ii) were found to have moderately higher levels of ROS than control cells (Fig. 2A, panel i). Increased levels of ROS were detected after $12 \mathrm{hr}$ of rotenone treatment (Fig. 2A, panel iv) and even higher levels after $24 \mathrm{hrs}$ of exposure to the toxin (Fig. 2A, panel vi). These results demonstrate that rotenone induces increased generation of ROS in S. pombe cells without inhibiting cellular respiration.

Similar to some types of mammalian cells, such as fibroblasts and neuronal cells [25;26], mitochondria in S. pombe undergo dynamic processes of fusion and fission to form tubular networks that are largely contiguous in appearance and which in interphase cells typically span the length of the cell from tip-to-tip [27;28]. Since rotenone has been shown to cause fragmentation of mitochondrial networks in mammalian cells [29;30], we sought to determine whether it has a similar effect on mitochondrial organization in S. pombe cells. To do this, mid-log phase cultures of wild type $S$. pombe cells were treated with rotenone for 4 hr, stained with the fluorescent mitochondrial dye MitoTracker Green FM (MTGreen), and visualized by fluorescence microscopy. In contrast to cells in control cultures, which exhibited a typical pattern of largely contiguous tubular mitochondrial networks (Fig. 2B, left panel), we observed that in cultures treated with rotenone, greater than $90 \%$ of cells exhibited complete or nearly complete fragmentation of mitochondrial organization (Fig. 2B, right panel). Thus, similar to its effects in mammalian cells, rotenone induces fragmentation of tubular mitochondrial networks in S. pombe cells.

\section{The MAP kinase Pmk1 and PKA are required for rotenone resistance in S. pombe}

Having determined that rotenone induces production of ROS in S. pombe cells, we investigated whether protein kinases involved or implicated in oxidative stress responses are required for rotenone resistance in this organism. S. pombe has two MAPKs, the p38 MAPK homolog Spc1 (a.k.a. Sty1 and Phh1) and the ERK-related MAPK Pmk1 (a.k.a. Spm1), that contribute differentially to oxidative stress responses [17;18;31;32;33;34]. Although the role of PKA in $S$. pombe oxidative stress response has not been clearly established, the gene encoding the PKA catalytic subunit, pkal [35], is induced by multiple extracellular stresses, including oxidative stress, and has been classified as a core environmental stress response (CESR) gene [36]. To determine whether $p m k 1 \Delta$, $\operatorname{spcl\Delta }$, or $p k a 1 \Delta$ mutants are hypersensitive to rotenone, mid-log phase cultures of the mutant strains and wild type $S$. pombe cells were serially diluted and tested for growth on medium containing rotenone and on control medium lacking the toxin. As shown in Fig. 3A, we found that the pmkl $1 \Delta$ and pkal $\Delta$ mutants were profoundly more sensitive to rotenone than wild type $S$. pombe cells, with the pkal $\Delta$ mutant being discernibly more sensitive to the toxin than the pmkl $\Delta$ mutant.

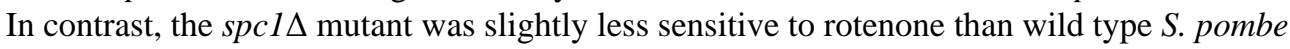
cells (Fig. 3A and B).

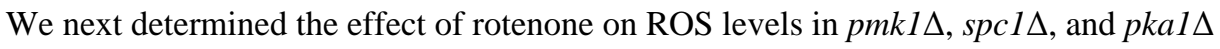
mutants. ROS levels were similar in control cultures of wild type (Fig. 3Ci) and pmkl $\Delta$ cells (Fig. 3Cii) and, by comparison, slightly reduced in spc1 $1 \Delta$ cells (Fig. 3Ciii). Interestingly, we found that ROS levels were markedly higher in control cultures of the pkal $\Delta$ mutant in comparison to the other strains (Fig. 3Civ). Consistent with results of growth assays 
described above, we found that rotenone induced only a very slight increase in ROS levels in spcl $\Delta$ cells (Fig. 3Cvii) relative to the increase detected in wild type cells (Fig. 3Cv). In contrast, rotenone induced levels of ROS production in both pmk1 $\Delta$ cells (Fig. 3Cvi) and pkal $\Delta$ cells (Fig. 3Cviii) that were dramatically higher than those induced in wild type cells. These findings suggest that physiological clearance of rotenone-induced ROS in S. pombe is at least partially dependent on Pmk1 and PKA but not on Spc1.

We next examined the effects of rotenone on mitochondrial organization in $p m k 1 \Delta, \operatorname{spcl\Delta }$, and pkal $\Delta$ cells. We found that in untreated cultures of all three strains, mitochondria were organized into largely contiguous tubular structures (Fig. 3D, panels i, iii, and v, respectively), suggesting that under normal culturing conditions, these protein kinases are not required for maintenance of tubular mitochondrial networks. We observed that rotenone induced marked fragmentation of mitochondrial organization in pmkl $\Delta$ cells (Fig. 3D, panels ii), albeit at a lower frequency ( $\approx 70 \%$ of cells) than that detected in cultures of wild type $S$. pombe cells (>90\% of cells). In contrast, rotenone had a marginal effect on mitochondrial organization in $s p c 1 \Delta$ cells, the majority of which retained largely contiguous tubular mitochondrial networks after rotenone treatment (Fig. 3D, panels vi). Interestingly, in pkal $\Delta$ cells treated with rotenone, mitochondria were found to be aggregated near the cell tips in about $75 \%$ of cells (Fig. 3D, panel v).

\section{Discussion}

We have shown here that rotenone, despite having no measurable effect on $S$. pombe respiration, nevertheless induces generation of ROS and disruption of tubular mitochondrial networks in $S$. pombe cells, two well documented effects of the toxin in mammalian cells [22]. Importantly, we have shown that two protein kinases, the ERK-related MAPK Pmk1 and PKA, are required for normal resistance to rotenone in $S$. pombe. In contrast, we showed that $S$. pombe cells lacking the $\mathrm{p} 38$ MAPK Spc1 exhibited modest resistance to rotenone. Our findings that MAPKs contribute to rotenone resistance in $S$. pombe are quite intriguing, given that previous studies have shown that ERK, p38, and JNK MAPKs are activated in neuronal tissues of PD patients and that ERK2 is associated with Lewy bodies, a hallmark of PD pathophysiology [37;38]. Contrary to its effects on at least some types of mammalian cells, we found that rotenone does not induce disruption of microtubules in treated S. pombe cells (Supplemental Online Data).

An unexpected finding of this study was that $S$. pombe PKA mutant cells have higher levels of ROS than wild type $S$. pombe cells under normal culturing conditions, suggesting that PKA plays a basal role in the elimination of ROS in S. pombe. It is possible that the elevated levels of ROS in pkal $\Delta$ cells might result in higher basal activity of oxidative stress response systems, including the Spc1 pathway, thus accounting for the previously described resistance of pkal $\Delta$ mutants to hydrogen peroxide stress and providing a possible explanation for the apparent antagonism of the cAMP-PKA pathway toward the Spc1 MAPK pathway, which has been suggested by previous genetic studies [39;40;41].

While this manuscript was in preparation, it was reported by Castro and coworkers that rotenone enhances cytotoxicity of the protein kinase inhibitor staurosporine in the budding yeast, S. cerevisiae, and the filamentous fungus, Neurospora crassa [42]. Although mitochondria of $N$. crassa have complex I, Castro et al. showed that rotenone enhances staurosporine toxicity even in complex I mutants of this fungus. The findings we have reported here together with the results of the Castro et al. study and those of Choi et al., who showed that rotenone is toxic to mouse dopaminergic neurons lacking complex I, make it unequivocally clear that rotenone has cytotoxic properties that are distinct from its inhibitory effects on complex I. The identification of molecular targets of and physiological responses 
to rotenone in model eukaryotes such as $S$. pombe, $S$. cerevisiae, and $N$. crassa may provide new insights into molecular processes that are relevant to the pathogenesis of PD.

\section{Supplementary Material}

Refer to Web version on PubMed Central for supplementary material.

\section{Acknowledgments}

The authors thank J. Henkel for technical assistance. This study was funded by National Institutes of Health grant R01GM068685 (to S.M.).

\section{References}

1. Soloway SB. Naturally occurring insecticides. Environ Health Perspect. 1976; 14:109-117. [PubMed: 789058]

2. Leonard JW. Notes on the Use of Derris as a Fish Poison. Transactions of the American Fisheries Society. 1939; 68:269-280.

3. Lummen P. Complex I inhibitors as insecticides and acaricides. Biochim Biophys Acta. 1998; 1364:287-296. [PubMed: 9593947]

4. Hirst J, Carroll J, Fearnley IM, Shannon RJ, Walker JE. The nuclear encoded subunits of complex I from bovine heart mitochondria. Biochim Biophys Acta. 2003; 1604:135-150. [PubMed: 12837546]

5. Brinkley BR, Barham SS, Barranco SC, Fuller GM. Rotenone inhibition of spindle microtubule assembly in mammalian cells. Exp Cell Res. 1974; 85:41-46. [PubMed: 4857086]

6. Srivastava P, Panda D. Rotenone inhibits mammalian cell proliferation by inhibiting microtubule assembly through tubulin binding. FEBS J. 2007; 274:4788-4801. [PubMed: 17697112]

7. Betarbet R, Sherer TB, MacKenzie G, Garcia-Osuna M, Panov AV, Greenamyre JT. Chronic systemic pesticide exposure reproduces features of Parkinson's disease. Nat Neurosci. 2000; 3:1301-1306. [PubMed: 11100151]

8. Uversky VN. Neurotoxicant-induced animal models of Parkinson's disease: understanding the role of rotenone, maneb and paraquat in neurodegeneration. Cell Tissue Res. 2004; 318:225-241. [PubMed: 15258850]

9. Schapira AH, Cooper JM, Dexter D, Jenner P, Clark JB, Marsden CD. Mitochondrial complex I deficiency in Parkinson's disease. Lancet. 1989; 1:1269. [PubMed: 2566813]

10. Mizuno Y, Ohta S, Tanaka M, Takamiya S, Suzuki K, Sato T, Oya H, Ozawa T, Kagawa Y. Deficiencies in complex I subunits of the respiratory chain in Parkinson's disease. Biochem Biophys Res Commun. 1989; 163:1450-1455. [PubMed: 2551290]

11. Schapira AH. Mitochondria in the aetiology and pathogenesis of Parkinson's disease. Lancet Neurol. 2008; 7:97-109. [PubMed: 18093566]

12. Langston JW, Forno LS, Rebert CS, Irwin I. Selective nigral toxicity after systemic administration of 1-methyl-4-phenyl-1,2,5,6-tetrahydropyrine (MPTP) in the squirrel monkey. Brain Res. 1984; 292:390-394. [PubMed: 6607092]

13. Choi WS, Kruse SE, Palmiter RD, Xia Z. Mitochondrial complex I inhibition is not required for dopaminergic neuron death induced by rotenone, MPP+, or paraquat. Proc Natl Acad Sci U S A. 2008; 105:15136-15141. [PubMed: 18812510]

14. Joseph-Horne T, Hollomon DW, Wood PM. Fungal respiration: a fusion of standard and alternative components. Biochim Biophys Acta. 2001; 1504:179-195. [PubMed: 11245784]

15. Gabaldon T, Rainey D, Huynen MA. Tracing the evolution of a large protein complex in the eukaryotes, NADH:ubiquinone oxidoreductase (Complex I). J Mol Biol. 2005; 348:857-870. [PubMed: 15843018]

16. Heslot H, Goffeau A, Louis C. Respiratory metabolism of a "petite negative"yeast Schizosaccharomyces pombe 972h. J Bacteriol. 1970; 104:473-481. [PubMed: 4394400] 
17. Zaitsevskaya-Carter T, Cooper JA. Spm1, a stress-activated MAP kinase that regulates morphogenesis in S.pombe. Embo J. 1997; 16:1318-1331. [PubMed: 9135147]

18. Toda T, Dhut S, Superti-Furga G, Gotoh Y, Nishida E, Sugiura R, Kuno T. The fission yeast pmk1+ gene encodes a novel mitogen-activated protein kinase homolog which regulates cell integrity and functions coordinately with the protein kinase C pathway. Mol Cell Biol. 1996; 16:6752-6764. [PubMed: 8943330]

19. Alfa, C.; Fantes, P.; Hyams, J.; McLeod, M.; Warbrick, E. Experiments with fission yeast: A laboratory course manual. Cold Spring Harbor, NY: Cold Spring Harbor Laboratory Press; 1993.

20. Huard S, Chen M, Burdette KE, Fenyvuesvolgyi C, Yu M, Elder RT, Zhao RY. HIV-1 Vprinduced cell death in Schizosaccharomyces pombe is reminiscent of apoptosis. Cell Res. 2008; 18:961-973. [PubMed: 19160543]

21. Degli Esposti M. Inhibitors of NADH-ubiquinone reductase: an overview. Biochim Biophys Acta. 1998; 1364:222-235. [PubMed: 9593904]

22. Li N, Ragheb K, Lawler G, Sturgis J, Rajwa B, Melendez JA, Robinson JP. Mitochondrial complex I inhibitor rotenone induces apoptosis through enhancing mitochondrial reactive oxygen species production. J Biol Chem. 2003; 278:8516-8525. [PubMed: 12496265]

23. Greenamyre JT, Betarbet R, Sherer TB. The rotenone model of Parkinson's disease: genes, environment and mitochondria. Parkinsonism Relat Disord. 2003; 9(Suppl 2):S59-S64. [PubMed: 12915069]

24. Bindokas VP, Jordan J, Lee CC, Miller RJ. Superoxide production in rat hippocampal neurons: selective imaging with hydroethidine. J Neurosci. 1996; 16:1324-1336. [PubMed: 8778284]

25. Barsoum MJ, Yuan H, Gerencser AA, Liot G, Kushnareva Y, Graber S, Kovacs I, Lee WD, Waggoner J, Cui J, White AD, Bossy B, Martinou JC, Youle RJ, Lipton SA, Ellisman MH, Perkins GA, Bossy-Wetzel E. Nitric oxide-induced mitochondrial fission is regulated by dynaminrelated GTPases in neurons. EMBO J. 2006; 25:3900-3911. [PubMed: 16874299]

26. Amchenkova AA, Bakeeva LE, Chentsov YS, Skulachev VP, Zorov DB. Coupling membranes as energy-transmitting cables. I. Filamentous mitochondria in fibroblasts and mitochondrial clusters in cardiomyocytes. J Cell Biol. 1988; 107:481-495. [PubMed: 3417757]

27. Chiron S, Gaisne M, Guillou E, Belenguer P, Clark-Walker GD, Bonnefoy N. Studying mitochondria in an attractive model: Schizosaccharomyces pombe. Methods Mol Biol. 2007; 372:91-105. [PubMed: 18314720]

28. Yaffe MP, Harata D, Verde F, Eddison M, Toda T, Nurse P. Microtubules mediate mitochondrial distribution in fission yeast. Proc Natl Acad Sci U S A. 1996; 93:11664-11668. [PubMed: 8876193]

29. Benard G, Bellance N, James D, Parrone P, Fernandez H, Letellier T, Rossignol R. Mitochondrial bioenergetics and structural network organization. J Cell Sci. 2007; 120:838-848. [PubMed: 17298981]

30. Plecita-Hlavata L, Lessard M, Santorova J, Bewersdorf J, Jezek P. Mitochondrial oxidative phosphorylation and energetic status are reflected by morphology of mitochondrial network in INS-1E and HEP-G2 cells viewed by 4Pi microscopy. Biochim Biophys Acta. 2008; 1777:834846. [PubMed: 18452700]

31. Millar JB, Buck V, Wilkinson MG. Pyp1 and Pyp2 PTPases dephosphorylate an osmosensing MAP kinase controlling cell size at division in fission yeast. Genes Dev. 1995; 9:2117-2130. [PubMed: 7657164]

32. Kato T Jr. Okazaki K, Murakami H, Stettler S, Fantes PA, Okayama H. Stress signal, mediated by a Hog1-like MAP kinase, controls sexual development in fission yeast. FEBS Lett. 1996; 378:207-212. [PubMed: 8557102]

33. Shiozaki K, Russell P. Cell-cycle control linked to extracellular environment by MAP kinase pathway in fission yeast. Nature. 1995; 378:739-743. [PubMed: 7501024]

34. Chen D, Wilkinson CR, Watt S, Penkett CJ, Toone WM, Jones N, Bahler J. Multiple pathways differentially regulate global oxidative stress responses in fission yeast. Mol Biol Cell. 2008; 19:308-317. [PubMed: 18003976] 
35. Maeda T, Watanabe Y, Kunitomo H, Yamamoto M. Cloning of the pka1 gene encoding the catalytic subunit of the cAMP-dependent protein kinase in Schizosaccharomyces pombe. J Biol Chem. 1994; 269:9632-9637. [PubMed: 8144551]

36. Chen D, Toone WM, Mata J, Lyne R, Burns G, Kivinen K, Brazma A, Jones N, Bahler J. Global transcriptional responses of fission yeast to environmental stress. Mol Biol Cell. 2003; 14:214229. [PubMed: 12529438]

37. Ferrer I, Blanco R, Carmona M, Puig B, Barrachina M, Gomez C, Ambrosio S. Active, phosphorylation-dependent mitogen-activated protein kinase (MAPK/ERK), stress-activated protein kinase/c-Jun N-terminal kinase (SAPK/JNK), and p38 kinase expression in Parkinson's disease and Dementia with Lewy bodies. J Neural Transm. 2001; 108:1383-1396. [PubMed: 11810403]

38. Nagao M, Hayashi H. Mixed lineage kinase 2 and hippocalcin are localized in Lewy bodies of Parkinson's disease. J Neurol Sci. 2009; 281:51-54. [PubMed: 19332348]

39. Stettler S, Warbrick E, Prochnik S, Mackie S, Fantes P. The wis1 signal transduction pathway is required for expression of cAMP- repressed genes in fission yeast. J Cell Sci. 1996; 109:19271935. [PubMed: 8832415]

40. Davidson MK, Shandilya HK, Hirota K, Ohta K, Wahls WP. Atf1-Pcr1-M26 complex links stressactivated MAPK and cAMP-dependent protein kinase pathways via chromatin remodeling of cgs2+ J Biol Chem. 2004; 279:50857-50863. [PubMed: 15448137]

41. Roux AE, Quissac A, Chartrand P, Ferbeyre G, Rokeach LA. Regulation of chronological aging in Schizosaccharomyces pombe by the protein kinases Pka1 and Sck2. Aging Cell. 2006; 5:345-357. [PubMed: 16822282]

42. Castro A, Lemos C, Falcao A, Fernandes AS, Glass NL, Videira A. Rotenone enhances the antifungal properties of staurosporine. Eukaryot Cell. 2010; 9:906-914. [PubMed: 20435699] 
A

+ Rotenone $(\mu \mathrm{g} / \mathrm{ml})$
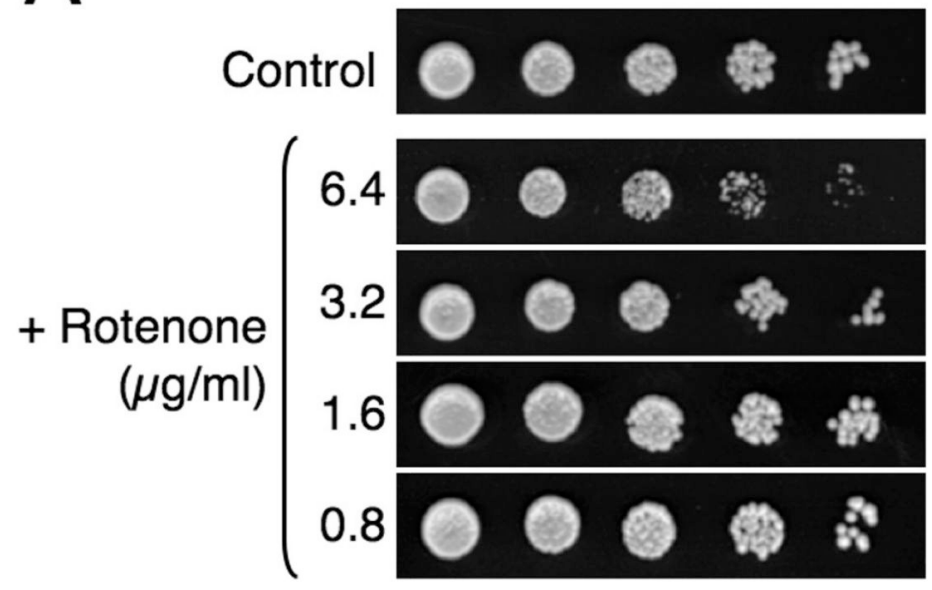

B

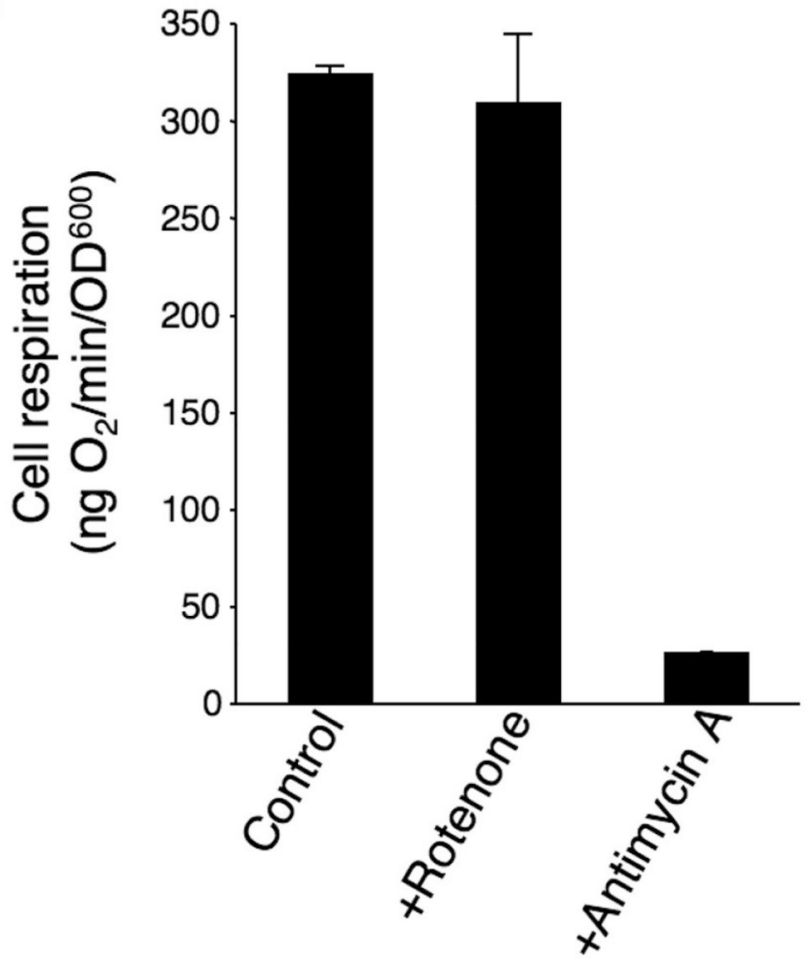

Fig. 1. Effects of rotenone on $S$. pombe cell growth and respiration

(A) Wild type $S$. pombe cells were cultured in YES medium to mid-log phase, harvested by centrifugation, and resuspended in YES at $5 \times 10^{6}$ cells $/ \mathrm{ml}$. The resulting cell suspension was serially diluted (1:5) and $3 \mu \mathrm{l}$ of each dilution was spotted onto YES control medium (top panel) or YES supplemented with rotenone at the indicated concentrations (lower panels) and the plates were incubated for 3 days at $30^{\circ} \mathrm{C}$. (B) Respiration rates of S. pombe control cultures and cultures treated with rotenone $(6.4 \mu \mathrm{g} / \mathrm{ml})$ or antimycin A $(1 \mu \mathrm{g} / \mathrm{ml})$ for $3 \mathrm{hr}$. 


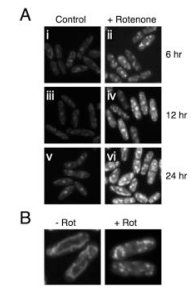

Fig. 2. Rotenone induces generation of ROS and fragmentation of mitochondrial networks in $S$. pombe cells

(A) $S$. pombe cells cultured to mid-log phase in YES medium were treated with rotenone $(6.4 \mu \mathrm{g} / \mathrm{ml})$ for 6,12 , or $24 \mathrm{hr}$, as indicated. Control cells were treated with a volume of DMSO equivalent to that used for the rotenone treated cells for the indicated lengths of time. Control cells (panels i, iii, and v) and rotenone treated cells (panels ii, iv, and vi) were stained with DHE and visualized by fluorescence microscopy for detection of ROS. (B) $S$. pombe cells were cultured to mid-log phase in YES and treated, or not, with rotenone (6.4 $\mu \mathrm{g} / \mathrm{ml}$ ) for $4 \mathrm{hr}$. Control cells (left panel) and rotenone treated cells (right panel) were stained with MTGreen and visualized by fluorescence microscopy. 


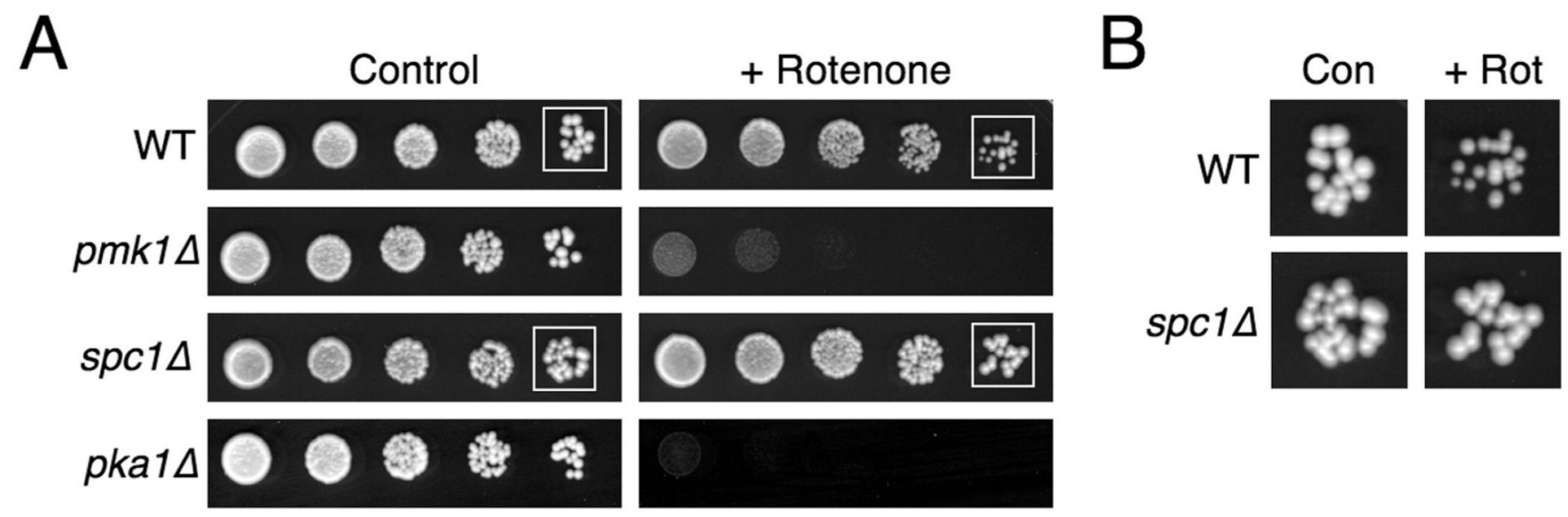

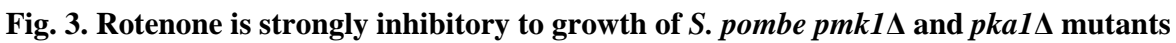
(A) Wild type, $p m k 1 \Delta$, $\operatorname{spc} 1 \Delta$, and pkal $\Delta$ cells were cultured and processed as described for Fig. 1A before spotting onto YES or YES supplemented with rotenone $(6.4 \mu \mathrm{g} / \mathrm{ml})$ and incubating the plates at $30^{\circ} \mathrm{C}$ for 3 days. (B) Magnified view of colonies from boxed spots in panel A of control (left panels) and rotenone treated (right panels) wild type (top panels) and spcl $1 \Delta$ strains (bottom panels). 


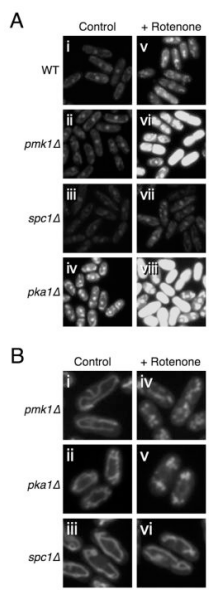

Fig. 4. Effects of rotenone on ROS levels and mitochondrial organization of $S$. pombe MAPK and PKA mutants

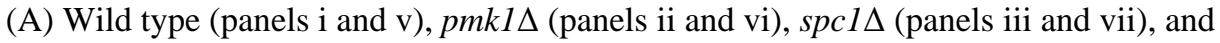
pkal $\Delta$ (panels iv and viii) S. pombe strains were cultured to mid-log phase in YES, treated (panels v-viii), or not (panels i-iv), with rotenone $(6.4 \mu \mathrm{g} / \mathrm{ml})$ for $12 \mathrm{hr}$, stained with DHE,

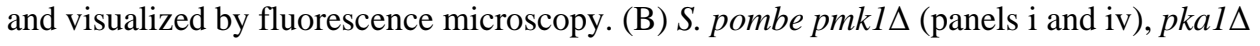
(panels ii and v), and $\operatorname{spcl\Delta }$ (panels iii and vi) strains were cultured to mid-log phase in YES medium, treated (panels iv-vi), or not (panels i-iii), with rotenone $(6.4 \mu \mathrm{g} / \mathrm{ml}$ ) for $4 \mathrm{hr}$, stained with MTGreen for detection of mitochondria, and visualized by fluorescence microscopy. 